Louisiana State University

LSU Digital Commons

Faculty Publications

Department of Physics \& Astronomy

4-10-2003

\title{
Gravitationally induced neutrino asymmetry
}

Parampreet Singh

Inter-University Centre for Astronomy and Astrophysics India

Banibrata Mukhopadhyay

Inter-University Centre for Astronomy and Astrophysics India

Follow this and additional works at: https://digitalcommons.Isu.edu/physics_astronomy_pubs

\section{Recommended Citation}

Singh, P., \& Mukhopadhyay, B. (2003). Gravitationally induced neutrino asymmetry. Modern Physics Letters A, 18 (11), 779-785. https://doi.org/10.1142/S0217732303009691

This Article is brought to you for free and open access by the Department of Physics \& Astronomy at LSU Digital Commons. It has been accepted for inclusion in Faculty Publications by an authorized administrator of LSU Digital Commons. For more information, please contact ir@lsu.edu. 


\title{
GRAVITATIONALLY INDUCED NEUTRINO ASYMMETRY
}

\author{
PARAMPREET SINGH*and BANIBRATA MUKHOPADHYAY ${ }^{\dagger}$ \\ Inter-University Centre for Astronomy and Astrophysics, \\ Post Bag 4, Ganeshkhind, Pune 411007, India
}

Received (02 December 2002)

Revised (10 February 2003)

\begin{abstract}
We study the propagation of Dirac neutrinos in gravitational backgrounds and show that the gravitational interaction can lead to neutrino asymmetry due to modifications in dispersion relation. We give some examples of spacetime geometries where such asymmetry can arise. This asymmetry would have contributed to the relic neutrino asymmetry through the interaction of neutrinos with primordial black holes before neutrinos decoupled. In the present epoch it may be generated in accretion around high temperature rotating black holes.
\end{abstract}

The propagation of test particles in gravitational backgrounds has yielded many interesting effects in both classical and quantum realms. If the test particle has a dipole moment then its motion deviates from the geodesic of a spin-free particle and is described by the Mathisson-Papapetrou equation ${ }^{1,2}$ which may also be extended to the case of particles with intrinsic spin ${ }^{3}$. There have been various further studies and extensions dealing with dynamics of such bodies in curved backgrounds at both classical and quantum mechanical level ${ }^{4}$.

In this work, we bring to the attention another manifestation of the above effect for the case of propagation of Dirac neutrinos in curved backgrounds and the resulting neutrino asymmetry. We would show that when mass eigenstates of Dirac neutrinos propagate in gravitational backgrounds, then depending upon the form of the background metric, a neutrino asymmetry can originate. In a recent paper ${ }^{5}$ we have highlighted this effect for the case of Kerr black holes in the present epoch. In this paper we would give a simple rule to identify when a background spacetime would yield a neutrino asymmetry. Apart from being generated in the present epoch, this asymmetry would also be generated in the early universe through the interaction of neutrinos with primordial black holes formed in QCD phase transition. We would also speculate about its relevance in future collider experiments.

*E-mail:param@iucaa.ernet.in

†E-mail:bm@iucaa.ernet.in 
The neutrino asymmetry as is well understood in the literature arises due to early Universe processes. If the baryon and lepton numbers are different in our Universe, then since our Universe is electrically neutral, it is argued that lepton asymmetry manifests itself in the form of neutrino asymmetry. Large lepton asymmetries can arise in early Universe through, for example, Affleck-Dine mechanism ${ }^{6-7}$. The gravitationally induced neutrino asymmetry would contribute to this early Universe asymmetry due to interaction of neutrinos with primordial black holes before the neutrinos decoupled at $t \sim 1 \mathrm{~s}$ after big-bang. Since relic neutrino asymmetry has important effects (and hence can be constrained) by big-bang nucleosynthesis and power spectrum of cosmic microwave background ${ }^{8}$.

One of the first effects for neutrinos in curved backgrounds was studied by Vilenkin ${ }^{9}$ where he sought for a macroscopic parity violation as an extension of Wu's celebrated experiment on Cobalt-60 confirming parity violation in nature. Vilenkin was able to show that for the case of a rotating black hole more neutrinos are emitted in the direction opposite to the angular momentum of the black hole, thus providing a hint that neutrinos in gravitational settings can provide interesting effects. This effect was further studied and related to pulsar velocities ${ }^{10-12}$. In more recent times effects of gravitational field on neutrino oscillations have also caught a lot of attention ${ }^{13-17}$.

As noted before, when a spinning test particle propagates in the curved spacetime, the coupling of its spin with the spin connection of the background field produces an interaction term. This interaction appears in a manner similar to the propagation of a test charged spinor in an electromagnetic field on a flat background 18. The spin connection in curved spacetime plays a similar role as of electromagnetic four-vector potential in the flat space. It is very interesting to note that the interaction term would not preserve CPT if the background spacetime contribution does not flip sign under CPT transformation. When the spinor under consideration is chosen as a Dirac neutrino, this interaction under CPT will give rise to opposite sign for a left-handed (neutrino) and right-handed (anti-neutrino) fields.

The general Dirac Lagrangian density in curved spacetime is given as

$$
\mathcal{L}=\operatorname{det}(e)\left(i \bar{\psi} \gamma^{a} D_{a} \psi-m \bar{\psi} \psi\right),
$$

where the covariant derivative and spin connection are defined as

$$
\begin{gathered}
D_{a}=\left(\partial_{a}-\frac{i}{4} \omega_{b c a} \sigma^{b c}\right), \\
\omega_{b c a}=e_{b \lambda}\left(\partial_{a} e_{c}^{\lambda}+\Gamma_{\gamma \mu}^{\lambda} e_{c}^{\gamma} e_{a}^{\mu}\right) .
\end{gathered}
$$

Here $\sigma^{b c}=\frac{i}{2}\left[\gamma^{b}, \gamma^{c}\right]$ is the generator of tangent space Lorentz transformation, the Latin and Greek alphabets indicate the flat and curved space coordinate respectively and we would work in units, $c=\hbar=k_{B}=1$ and with signature (+---). 
The spin connection term in the Dirac Lagrangian will give rise to a combination of vector, $\bar{\psi} A_{a} \gamma^{a} \psi$ and an axial-vector, $\bar{\psi} B^{d} \gamma^{5} \gamma_{d} \psi$ coupling. The vector term is anti-hermitian and disappears when the hermitian conjugate part of Lagrangian is added to (1). The only interaction which survives in $\mathcal{L}$ is the axial-vector part and (1) reduces to

$$
\mathcal{L}=\operatorname{det}(e) \bar{\psi}\left[\left(i \gamma^{a} \partial_{a}-m\right)+\gamma^{a} \gamma^{5} B_{a}\right] \psi=\mathcal{L}_{f}+\mathcal{L}_{I},
$$

where,

$$
B^{d}=\epsilon^{a b c d} e_{b \lambda}\left(\partial_{a} e_{c}^{\lambda}+\Gamma_{\alpha \mu}^{\lambda} e_{c}^{\alpha} e_{a}^{\mu}\right) .
$$

If $B^{d}$ does not change sign under CPT transformation then $\mathcal{L}_{I}$ is odd under it. The important factor for our application is that the axial interaction term $\left(\mathcal{L}_{I}\right)$ has different signs for left and right chiral fields and for neutrino, $\psi$, and anti-neutrino, $\psi^{c}$, and it may be expressed as

$$
\begin{gathered}
\bar{\psi} \gamma^{a} \gamma^{5} \psi=-\bar{\psi}_{L} \gamma^{a} \psi_{L}, \\
\bar{\psi}^{c} \gamma^{a} \gamma^{5} \psi^{c}=\left(\bar{\psi}^{c}\right)_{R} \gamma^{a}\left(\psi^{c}\right)_{R}
\end{gathered}
$$

The corresponding dispersion relation for left and right chirality fields is given as

$$
\left(p_{a} \pm B_{a}\right)^{2}=m^{2},
$$

where the upper sign corresponds to particle and the lower sign is for anti-particle.

In the case of Dirac neutrino family, the particle is left-handed and its antiparticle partner is right-handed, hence expanding out (8), one can obtain the dispersion relation for left-handed (neutrino) and right-handed (anti-neutrino) fields as

$$
E_{\nu, \bar{\nu}}=\sqrt{\mathbf{p}^{2} \pm 2 B_{a} p^{a}+B_{a} B^{a}-m^{2}}
$$

where the $B_{a} p^{a}$ interaction term comes with a positive sign for the neutrino and with a negative sign for the anti-neutrino.

Note that $B_{a}$ depends on the metric and its derivative. Above dispersion relation thus tells us that there may be a split in energies of neutrinos and anti-neutrinos when they propagate on a curved background. Let us consider a situation in which beams of neutrinos and anti-neutrinos are propagating in a region of strong gravitational field. If $P_{i}$ and $P_{f}$ refer to two points of the interval over which asymmetry is measured, the asymmetry would be

$$
\Delta n=\frac{g}{(2 \pi)^{3}} \int_{P_{i}}^{P_{f}} d V \int d^{3} \mathbf{p}\left[\frac{1}{1+\exp \left(E_{\nu} / T\right)}-\frac{1}{1+\exp \left(E_{\bar{\nu}} / T\right)}\right]
$$


It is important to note that this asymmetry is independent of the choice of coordinates, which can be realized from the invariant form of the interaction term in the Lagrangian. Hence for simplicity, without any loss of generality, we would make the choice of Cartesian coordinates. In this coordinate system, we note that unless $B_{0}$ is non zero, the above integral is odd and hence asymmetry vanishes. Hence working in Cartesian coordinates, we find that a necessary condition to ensure a non zero neutrino asymmetry is that background metric should be such that $B_{0} \neq 0$. As $B_{0}$ is an axial-scalar potential, it is clear from its definition that it would be non vanishing only when one of the $g_{x y}, g_{y z}$ and $g_{x z}$ components is non zero. Similarly, one can find out under what conditions other components of this axial vector would be non vanishing.

If we write the most general metric for the background spacetime,

$$
d s^{2}=g_{00} d t^{2}-g_{i i} d x^{i} d x^{i}-2 g_{0 i} d t d x^{i}-g_{i j} d x^{i} d x^{j}
$$

and separate out the diagonal, spatio-temporal and spatial-spatial components,

Metric $=\underbrace{(\text { Diagnol Part })}_{I}+\underbrace{(\text { Spatio }- \text { Temporal Part })}_{I I}+\underbrace{(\text { Spatial }- \text { Spatial Part })}_{I I I}$

then if $I I$ and $I I I$ are identically zero then $B_{\mu}$ vanishes completely. In Cartesian coordinates, neutrino asymmetry would arise if and only if $I I I$ is non vanishing. Given a background spacetime, one can write it in Cartesian form and easily predict whether such a spacetime would give rise to neutrino asymmetry.

We now give some examples highlighting above rule. Let us first take the case of metric of a rotating black hole of mass $M$ and angular momentum $\vec{J}=M \vec{a}$, in the asymptotic limit i.e. $1 / r \rightarrow 0$ and neglecting terms of the order of $\left(r_{g} / r\right)^{3}$ and higher order, where $r_{g}=2 M / M_{\text {Planck }}^{2}$. The spacetime under this approximation is given by 19

$d s^{2}=\left(1-\frac{r_{g}}{r}+\left(\frac{r_{g}}{2 r}\right)^{2}\right) d t^{2}+2 \frac{r_{g}}{r^{3}} \epsilon_{j k l} a^{k} x^{l} d t d x^{j}-\left(1+\frac{r_{g}}{r}+\frac{1}{2}\left(\frac{r_{g}}{r}\right)^{2}\right) \delta_{i j} d x^{i} d x^{j}$

Since, all of the space-space cross terms, i.e. III in (12), are vanishing hence $B_{0}$ would be zero and thus the metric above for the rotating black hole in this approximation would not yield a non zero neutrino asymmetry.

Let us now consider Kerr metric in Cartesian coordinates without invoking any approximation. The spacetime interval is given by ${ }^{20}$

$$
\begin{aligned}
d s^{2} & =d t^{2}-d x^{2}-d y^{2}-d z^{2} \\
& -\frac{2 M r^{3}}{r^{4}+a^{2} z^{2}}\left[d t-\frac{1}{r^{2}+a^{2}}(r(x d x+y d y)+a(x d y-y d x))-\frac{z}{r} d z\right]^{2}
\end{aligned}
$$

${ }^{a}$ This happens because if $B_{0}=0$, then under $p_{i} \rightarrow-p_{i}, E_{\nu}=E_{\bar{\nu}}$. 
where $r$ is defined through,

$$
r^{4}-r^{2}\left(x^{2}+y^{2}+z^{2}-a^{2}\right)-a^{2} z^{2}=0 .
$$

The metric yields non vanishing space-space cross terms and hence the neutrino asymmetry ${ }^{5}$.

Another example of a spacetime where this asymmetry can occur is in the FRW metric with primordial tensor perturbations ${ }^{21}$,

$$
d s^{2}=S(\tau)^{2}\left[(1+2 \phi) d \tau^{2}-\alpha_{i} d x^{i} d \tau-\left((1+2 \beta) \delta_{i j}+h_{i j}\right) d x^{i} d x^{j}\right] .
$$

Here $S(\tau)$ is the expansion factor, $\phi$ and $\beta$ are scalar, $\alpha_{i}$ is vector and $h_{i j}$ is tensor fluctuation of the metric. Since the space-space cross terms are present one expects neutrino asymmetry in the case of this background spacetime.

We now consider a background spacetime with the parameters (for e.g. in the case of a Kerr black hole, mass and angular momentum) tuned in such a way that $\vec{B} \cdot \vec{p} \ll B_{0} p^{0}$ and $B_{a} B^{a} \ll 1$, then in the ultra-relativistic regime we get from (10),

$$
\Delta n=\frac{g}{2 \pi^{2}} \int_{P_{i}}^{P_{f}} d V \int_{0}^{\infty} T^{3}\left[\frac{1}{1+e^{u} e^{B_{0} / T}}-\frac{1}{1+e^{u} e^{-B_{0} / T}}\right] u^{2} d u
$$

where $u \equiv \mathbf{p} / T$. If $B_{0} \ll T$, then

$$
\Delta n \sim g T^{3}\left(\frac{\bar{B}_{0}}{T}\right)
$$

where $\bar{B}_{0}$ is $B_{0}$ integrated over the path from $P_{i}$ to $P_{f}$. The sign of this asymmetry depends on various parameters of the background metric. The asymmetry becomes significant for high temperatures.

This process would have made a contribution to the relic neutrino asymmetry in the period between $t \sim 10^{-5} \mathrm{~s}$, the time after big bang when QCD phase transitions occurred and primordial black holes were formed, and $t \sim 1 \mathrm{~s}$, the time of neutrino decoupling. The temperature of neutrinos during QCD phase transitions was of the order of $100 \mathrm{MeV}$ and went down to $1 \mathrm{MeV}$ when they decoupled. In a generic physical scenario, primordial black holes would be rotating and we can estimate the contribution to the relic asymmetry. Assuming that $\bar{B}_{0} \sim 10^{-5} \mathrm{erg}$ for a single such black hole the estimated asymmetry from (18) turns out to be $\Delta n \sim 10^{-13}$ in the period of QCD phase transitions. Similarly, at the epoch of neutrino decoupling this asymmetry would reduce to $\Delta n \sim 10^{-19}$ for $\bar{B}_{0} \sim 10^{-7} \mathrm{erg}$.

In the present epoch this asymmetry can become significant wherever there exist neutrinos with high temperature in strong gravitational fields with metrics deviating from spherical symmetry. One such arena would be for the neutrinos formed in Hawking radiation process of light mass black holes which will be at high 
temperature. ${ }^{b}$ For example a primordial black hole with mass $\gtrsim 10^{15} \mathrm{~g}$ would still exist today $^{c}$ and would have the Hawking temperature of $\gtrsim 10^{11} \mathrm{~K} \sim 10^{-5} \mathrm{erg}$. When the neutrinos come out from these black holes they will have the temperature as of $\gtrsim 10^{-5}$ erg even and may accrete around the black hole. If $\bar{B}_{0}$ is chosen of the order of $10^{-6}$ erg then the resulting neutrino asymmetry arising due to the accretion process of such a single black hole would be of the order of $10^{-16}$. With large number of such phenomena adding together, this asymmetry would increase.

Such an effect can also leave signatures at future collider experiments. If there exist large extra dimensions then the fundamental Planck scale can be around $\mathrm{TeV}$ ${ }^{23,24}$. In such a setting, parton-parton collisions would produce black holes with mass equal to the center of mass energy of two partons, if the impact parameter is smaller than the Schwarzschild radius ${ }^{25,26}$. The Large Hadron Collider experiment would touch proton-proton center of mass energies to about $14 \mathrm{TeV}$ and hence produce a copious amount of mini black holes which would have Hawking temperature of about $\sim 10^{26} \mathrm{~K}$ and would radiate out to standard model particles in $10^{-26} \mathrm{~s}$. If the black holes are assumed to be spinning and provided the flux of neutrinos is large enough then such an experiment could be an interesting setting for the study of gravitationally induced neutrino asymmetry, which can be pursued in future.

We thank D. V. Ahluwalia and N. Dadhich for various helpful discussions and suggestions. We would also wish to thank P. Dasgupta, A. Gupta, A. S. Majumdar, P. Mitra, S. Mohanty, T. Roy Choudhury and M. Sami for comments and useful discussions. PS thanks Council for Scientific and Industrial Research for grant number: 2-34/98(ii)E.U.-II.

\section{References}

1. M. Mathisson, Acta. Phys. Polon. 6, 167 (1937).

2. A. Papapetrou, Proc. Roy. Soc. Lond. A 209, 248 (1951).

3. J. Anandan, Il. Nuovo. Cim. A 53, 221 (1979).

4. For a review on earlier attempts on extension of Mathisson-Papapetrou equation for higher multipole moments and its extension see, W. G. Dixon, Phil. Tran. Roy. Soc. Lond. A 277, 59 (1974). Recently this problem has been studied through variational principle technique in J. Anandan, N. Dadhich, P. Singh, "Action principle formulation for motion of extended bodies in General Relativity," gr-qc/0212130. For a study of spinning particles on gravitational backgrounds see for eg., V. B. Bezerra, Phys. Rev. D35, 2031 (1987), J. Anandan, Phys. Lett. A 195, 284 (1994) and Y. N. Obukhov, Fortsch. Phys. 50, 711 (2002).

5. B. Mukhopadhyay, P. Singh, "Black holes as a source of neutrino degeneracy in the Universe," gr-qc/0301002.

${ }^{b}$ We thank D. V. Ahluwalia for discussions on this issue.

${ }^{c}$ However, the evaporation rate of black holes would be slower if we live on a brane universe ${ }^{22}$ and hence black holes with even larger mass might exist today. 
6. I. Affleck, M. Dine, Nucl. Phys. B 249, 361 (1985).

7. J. McDonald, Phys. Rev. Lett 16, 748 (2000).

8. M. Orito et al., Phys.Rev. D65 123504, (2002); A. Dolgov et al., Nucl. Phys. B 632, 363 (2002). For a review see, A. D. Dolgov, Phys. Rep. 370, 333 (2002).

9. A. Vilenkin, Phys. Rev. D20, 1807 (1979).

10. A. Kusenko, G. Segre, Phys. Rev. Lett. 77, 4872 (1996).

11. E. Nardi, J. I. Zuluaga, Ap. J. 549, 1076 (2001).

12. M. Barkovich, H. Casini, J. C. D'Olivo, R. Montemayor, Phys. Lett. B 506, 20 (2001).

13. D. V. Ahluwalia, C. Burgard, Gen. Rel. Grav. 28, 1161 (1996); Erratum: Gen. Rel. Grav. 29, 681 (1997).

14. D. Piriz, M. Roy, J. Wudka, Phys. Rev. 54, 1587 (1996).

15. D. V. Ahluwalia, Gen. Rel. Grav. 29, 1491 (1997).

16. K. Konno, M. Kasai, Prog. Theor. Phys. 100, 1145 (1998).

17. J. Wudka, Phys. Rev. D64, 065009 (2001).

18. B. Mukhopadhyay, Class. Quantum Grav. 17, 2017 (2000).

19. Z. Lalak, S. Pokorski, J. Wess, Phys. Lett. B 355, 453 (1995).

20. S. Chandrashekhar, "The Mathematical Theory of Black Holes," Oxford Univ. Press (1992).

21. S. Mohanty, B. Mukhopadhyay, A. R. Prasanna, "Baryogenesis from primordial tensor perturbations," hep-ph/0204257.

22. A. S. Majumdar, Phys. Rev. Lett. 90, 031303, (2003).

23. N. Arkani-Hamed, S. Dimopoulos, G. Dvali, Phys. Lett. B 429, 263 (1998).

24. N. Arkani-Hamed, S. Dimopoulos, Phys. Rev. D59, 086004 (1999).

25. S. Dimopoulos, G. Landsberg, Phys. Rev. Lett. 87, 161602, (2001).

26. S. B. Giddings, S. Thomas, Phys. Rev. D65, 056010 (2002). 